Pflegesoftware App für Pflegekräfte

— Die Formulierungshilfen von Standard Systeme, das bekannte Nachschlagewerk für Pflegefachkräfte, ist ab sofort auch als praktische App für Android- und AppleEndgeräte verfügbar. Unkompliziert installiert auf dem Handy oder Tablet haben Mitarbeiterinnen und Mitarbeiter in der Pflege ab sofort jederzeit mobil Zugriff auf die bewährten Inhalte aus der Printversion der Formulierungshilfen, wie Ressourcen, Probleme, Maßnahmen und Hilfsmittel. Auch die erst in der 2016-Version zusätzlich aufgenommenen Kriterien zur pflegefachlichen Risiko-Einschätzung im Rahmen der Ablaufschemata gemäß der Expertenstandards finden sich in der App-Version der Formulierungshilfen wieder und unterstützen das Pflegepersonal bei der täglichen Arbeit. Die App ist ab sofort sowohl im Google Play Store (für Android-Geräte) sowie im iTunes App Store (für Apple-Geräte) zum Preis von $14,99 €$ erhältlich.

www.standardsysteme.de

\section{Luftreiniger}

\section{Keimfreie Raumluft}

- Für einen heilungstherapeutischen Fortschritt spielt das Wohlbefinden des Patienten in den Therapieräumen eine wesentliche Rolle. Dabei ist die Luftqualität ein nicht zu vernachlässigbarer Faktor. Allerdings wird die Luftbeschaffenheit durch ein komplexes Zusammenspiel aus verschiedenen, oft schädlichen Bestandteilen bestimmt. Häufig schweben unangenehme Gerüche von vorherigen Behandlungssitzungen im Raum, ganz zu schweigen von Schadstoffen wie Viren oder Keime. Die Lösung für reine, keimfreie Luft, die das Wohlbefinden steigert, heißt AL-KO Pure. Das Gerät vernichtet nahezu vollständig bakterielle und virale Krankheitserreger und setzt dabei im Gegensatz zu vielen herkömmlichen Luftreinigern kein Ozon frei. Vielmehr wird die Ozonkonzentration in der Raumluft sogar reduziert. In therapeutischen Praxen stammen giftige Dämpfe zum Beispiel von Therapiegeräten. Außerdem senkt die Drei-Wege-Patrone den Ozon-Gehalt der Luft und verringert die

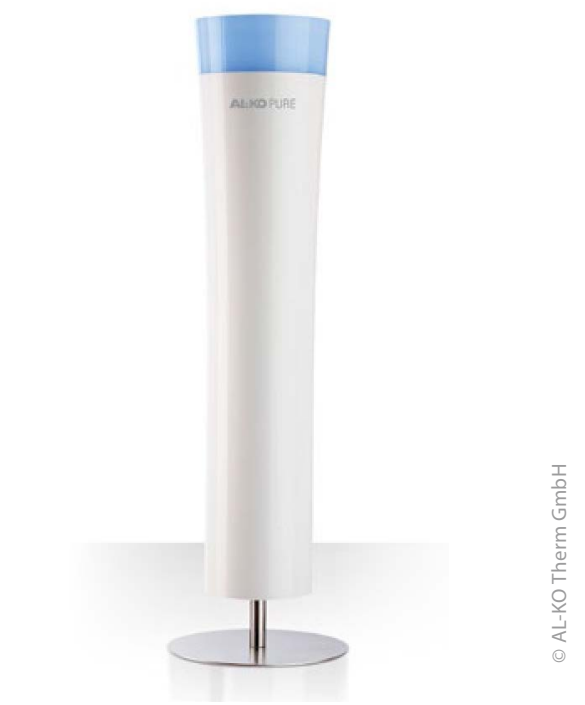

Gerüche im Raum, beispielsweise von Desinfektionsmitteln oder Schweißgeruch. Ein Luftsensor informiert zudem stets über die vorhandene Luftqualität, die sich durch einen Fingertipp intuitiv regeln lässt.

www.al-ko.com/de/pure/

\title{
Ulcus cruris venosum
}

\section{Kompressionsmaßnahmen sind der Tragpfeiler der Therapie}

— Grundlage einer erfolgreichen Behandlung von Beinulzera ist die adäquate Kompressionstherapie. Laut Prof. Dr. Knut Kröger, Krefeld, führt die Kompression kurzfristig zu einer besseren Sauerstoffversorgung der Gewebe und langfristig zur Vermeidung des trophischen Gewebeumbaus und damit zur Abheilung der venösen Ulzera. „Zahlreiche Untersuchungen belegen jedoch, dass die Kompression bei der Ulcus-Therapie im Praxisalltag zu selten eingesetzt wird", berichtete der Angiologe.

Stadienangepasste Kompressionsbehandlung: Beim Ulcus cruris venosum sei es überdies wichtig, so Kröger, dass sich die Kompressionstherapie an der aktuellen Wundsituation des Patienten orientiert. Unterschieden werden eine erste Phase, in der mit der Entstauungstherapie sowie der Wundbehandlung begonnen wird, eine Übergangsphase, in der die Ödemreduktion und Wundversorgung fortgesetzt werden und eine Erhaltungsphase, die der Rezidivprophylaxe gewidmet ist. „Die Stärke der Kompression muss immer dem Behandlungsziel entsprechen und zugleich an die Behandlungsphase angepasst sein", forderte Kröger.

Für die Therapie mit Kompressionsverbänden stehen Binden mit unterschiedlichen Eigenschaften hinsichtlich Kraft und Dehnung zur Verfügung. „In Deutschland hat die Kompressionstherapie mit Kurzzugbandagen, die ein geringes Dehnungsvermögen besitzen, eine lange Tradition", so Kröger. Sie erzeugen einen hohen Arbeitsdruck, setzen der Muskulatur bei Bewegung also einen hohen Widerstand entgegen und beeinflussen daher auch krankhafte Veränderungen in tieferliegenden Venen günstig.
Für die Kompressionstherapie von venösen Ulzera sind alternativ zu den Kurzzugbinden auch fertige Bindensysteme und inzwischen auch Kompressionsbandagen-Systeme wie circaid $^{\circledR}$ juxtacures $^{\circledast}$ verfügbar. Zu den Vorteilen dieses Systems zählt seine einfache Handhabung. Das Anlegen der Versorgung ist so unkompliziert, dass diese Aufgabe leicht vom Patienten selbst übernommen werden kann. Das circaid ${ }^{\circledR}$ Built-In-Pressure ${ }^{\circledR}$ system erlaubt dem Patienten das eigenständige Einstellen des verordneten Kompressionsniveaus und dessen Nachjustierung an sich verändernde Beinumfänge.

(wed)

Workshop "Abschied vom Ulcus in drei Schritten" anlässlich der practica Bad Orb, 23.10.2015 (Veranstalter: medi GmbH \& Co. $\mathrm{KG})$ 